\title{
Coherent Smith-Purcell radiation for minimally invasive bunch length measurement at the subpicosecond time scale
}

\author{
Philipp Heilø, ${ }^{*}$ Kurt Aulenbacher, Christoph Matejcek, Simon Friederich $\odot$, \\ Max Brukerø, ${ }^{\dagger}$ and Frank Fichtner \\ Institute for Nuclear Physics, Johannes Gutenberg University Mainz, Germany, \\ Helmholtz Institute Mainz, Germany \\ and GSI Helmholtzzentrum für Schwerionenforschung, Darmstadt, Germany
}

(Received 3 February 2021; accepted 5 April 2021; published 20 April 2021)

\begin{abstract}
We have designed a tool to measure the bunch length of an electron beam in a minimally invasive way by means of coherent Smith-Purcell radiation (SPR). The technique has been employed successfully at a test apparatus for the Mainz Energy-recovery Superconducting Accelerator MESA, demonstrating it is possible to determine the bunch length while losing less than $0.6 \%$ of the electron beam. The impact of the space charge on the bunch length can be reduced while tuning the longitudinal bunch preparation system during a live measurement at beam currents up to $1 \mathrm{~mA}$. Doing so, RMS bunch lengths of $70 \mu \mathrm{m}$ can be achieved in a typical operating mode of the low-energy beam transport system of MESA. In addition to the bunch length measurements, typical properties of the generated SPR are demonstrated.
\end{abstract}

DOI: 10.1103/PhysRevAccelBeams.24.042803

\section{INTRODUCTION}

In order to investigate ultrafast physical processes, short pulses of particles have been receiving more and more attention. In accelerator physics, short electron bunches are needed to ensure acceleration of the beam without losing too many particles or, in other words, to match the accelerator acceptance. In preparation for the construction of the Mainz Energy-recovering Superconding Accelerator (MESA), we have built a test apparatus comprising the first ten meters of the low-energy beam transport system at $100 \mathrm{keV}$ of MESA. As the longitudinal bunch preparation system is installed here, this apparatus is suitable for bunch length measurements, even at high beam currents. In those circumstances, Coulomb interactions between the electrons lead to a blow-up of the whole bunch (space charge).

\section{GENERATION OF COHERENT SPR}

If a charged particle passes by a metallic grating in close proximity, its evanescent field excites multiple resonant modes in the groove of the grating profile. These modes emit plane waves above the surface of the grating. An

\footnotetext{
*phheil@uni-mainz.de

Present address: Thomas Jefferson National Accelerator Facility, Newport News, Virginia 23606, USA.

Published by the American Physical Society under the terms of the Creative Commons Attribution 4.0 International license. Further distribution of this work must maintain attribution to the author(s) and the published article's title, journal citation, and DOI.
}

approach to calculating the power of the emitted SPR by solving Maxwell's equations with the periodic boundary conditions of the grating is described in the papers by van den Berg ([1] and [2]). Figure 1 shows a schematic sketch of the electron beam passing a grating at the distance $z_{0}$. For semirelativistic particles $(\beta=0.548)$ the wavelength $\lambda$ of the emitted radiation is in the order of magnitude of the grating period $D$ and is dependent on the observation angle $\eta$ and the order of diffraction $n[3]$.

$$
\lambda=\frac{D}{n}\left(\frac{1}{\beta}-\sin \eta\right)
$$

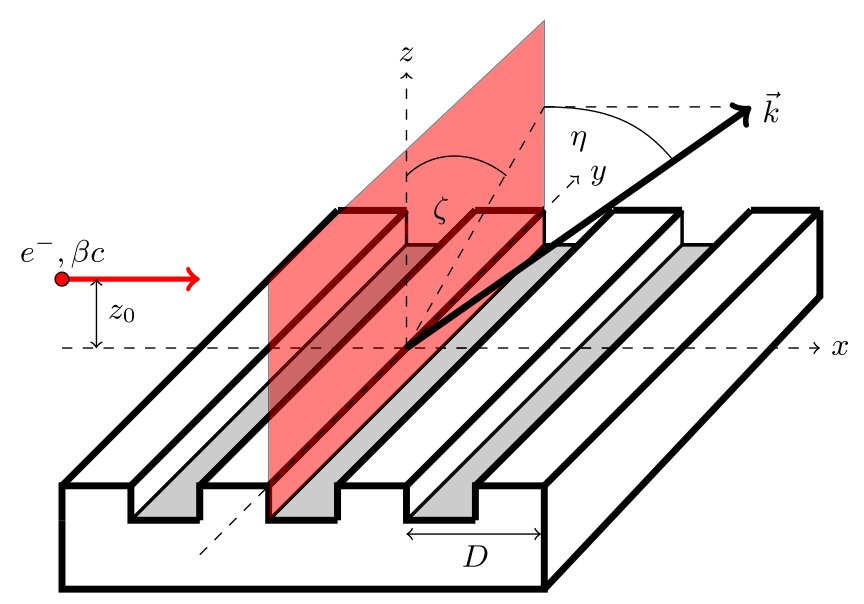

FIG. 1. Arrangement to produce SPR with corresponding coordinates and angles. $\vec{k}$ is the vector where SPR is observed. 
The wavelength is not dependent on the azimuth angle $\zeta$. The power of the emitted radiation per solid angle can be calculated using van den Berg's approach [4].

$$
\begin{aligned}
\frac{\mathrm{d} P}{\mathrm{~d} \Omega}= & \frac{I N_{w} e|n|^{2} \beta^{3} \cos ^{2} \eta \cos ^{2} \zeta}{2 D \epsilon_{0}(1-\beta \sin \eta)^{3}} \cdot\left|R_{n}(\eta, \zeta)\right|^{2} \cdot\left[S_{\mathrm{inc}}(\eta, \zeta)\right. \\
& \left.+\left(N_{e}-1\right) S_{\mathrm{coh}}(\eta, \zeta)\right]
\end{aligned}
$$

where $N_{w}$ is the number of grating periods, $I$ is the beam current, $e$ the elementary charge, $\epsilon_{0}$ the vacuum permittivity, $R_{n}$ a reflection coefficient dependent on the geometry of the grating, $N_{e}$ the number of electrons per bunch, and $S_{\text {inc }}, S_{\text {coh }}$ the coherent and incoherent part of the radiation, respectively. $S_{\text {coh }}$ is dependent on the charge distribution $f_{z}(z), f_{y}(y)$ and $f_{x}(x)$ of the electron bunch in space. The distribution in the longitudinal direction $f_{x}(x)$ can be expressed in terms of time $f_{t}(t)$ with the known velocity of the particles $\beta c$.

$$
\begin{aligned}
S_{\text {coh }}= & \left|\int_{0}^{\infty} \exp \left[-\frac{z}{2 h_{\text {int }}(\eta, \zeta)}\right] f_{z}(z) \mathrm{d} z\right|^{2} \\
& \times \cdot\left|\int_{-\infty}^{\infty} e^{-i k_{y} y} f_{y}(y) \mathrm{d} y\right|^{2} \cdot\left|\int_{-\infty}^{\infty} e^{-i \omega t} f_{t}(t) \mathrm{d} t\right|^{2},
\end{aligned}
$$

where the first term is governed by the ratio of the distance of the beam center from the grating surface and the interaction length $h_{\text {int }}$ :

$$
h_{\text {int }}(\eta, \zeta)=\frac{D(1 / \beta-\sin \eta)}{4 \pi|n| \sqrt{1 / \beta^{2}-1+\cos ^{2} \eta \sin ^{2} \zeta}}
$$

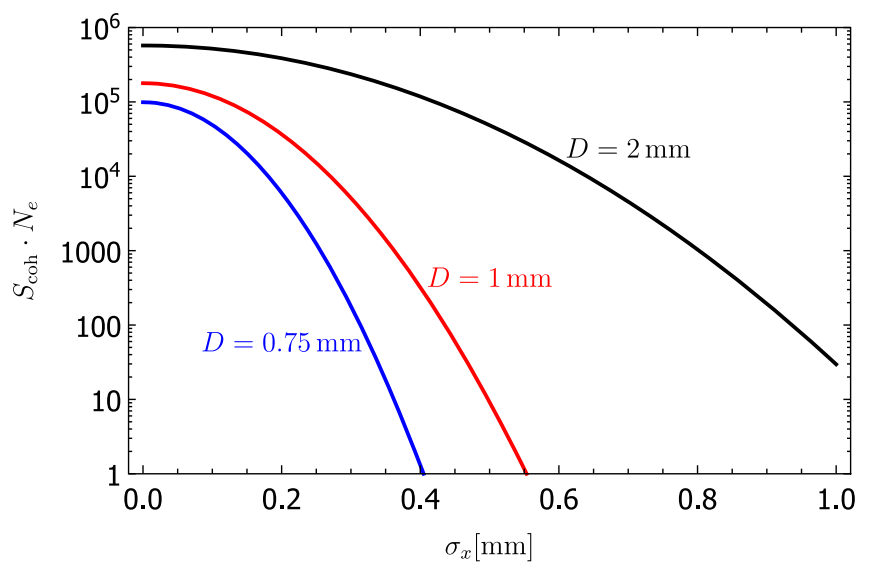

FIG. 2. Coherent enhancement factor $N_{e} \cdot S_{\text {coh }}$ for different bunch lengths $\sigma_{x}=\beta c \sigma_{t}$ and grating periods $D$ in the first radiation order $(n=1)$. Coherent SPR can only be observed if the bunch length $\sigma_{x}$ is smaller or comparable to half the grating period $D$. These curves show the effect of different values of $h_{\text {int }}(D)$, while the charge distribution of the beam is the same in each case.
The second term is approximately equal to one because the transverse size of the beam is much smaller than the width of the grating. The third term is defined by the charge distribution in the longitudinal direction $f_{t}(t)$, where $\omega=$ $2 \pi c / \lambda$ and $c$ is the speed of light in vacuum. We assume the charge distributions $f_{t}(t)$ and bunch lengths $\sigma_{t}$ to be Gaussian. For $\beta=0.548, S_{\mathrm{coh}}$ becomes significant and approaches one for $\sigma_{t} \beta c \leq D / 2$, and a coherent enhancement of the radiation by a factor of $N_{e}$ occurs. Figure 2 shows $N_{e} \cdot S_{\text {coh }}$ for a beam current of $I=1 \mathrm{~mA}$, corresponding to a particle number of $N_{e}=5 \times 10^{6}$. In this experiment, the incoherent part of the SPR cannot be observed because its intensity is below the background level; only coherent SPR can be observed, the power of which is in the order of $\mathrm{pW}$ to $\mathrm{nW}$. Because coherent SPR is emitted only if the bunch length $\sigma_{x} \lesssim D / 2$, placing gratings with different periods $D$ next to the beam yields an upper bound of the bunch length. A comparison of the calculated power with the measured one gives even more precise results.

\section{BEAM LINE SETUP AND PARAMETERS}

An important part of the new accelerator MESA is the low-energy beam transport system MELBA (Mesa lowenergy beam apparatus) connecting the $100 \mathrm{keV}$ electron source with the injector accelerator. Here, spin manipulation and bunch preparation for the injector take place. Due to the low energy, space charge is a challenging issue. A test setup has been built to check the functionality of devices and compare the beam parameters with the simulation [5-7]. The overall layout of the setup is shown in Fig. 3.

The electron beam is generated by emitting electrons from a $\mathrm{GaAs}$ photocathode using laser radiation at a wavelength of $800 \mathrm{~nm}$ and accelerating them with a DC voltage of $100 \mathrm{kV}$ [8]. In this experiment, the laser operates in DC mode and thus provides a continuous electron beam. The following beam line contains the longitudinal bunch preparation system, which converts this DC beam into short electron bunches using a system of two circularly deflecting $1.3 \mathrm{GHz}$ chopper cavities and a system of first- and second-harmonic buncher cavities. This system is based on that employed at MAMI [9] and adapted to the MESA rf frequency. With two buncher cavities, the injector can make use of a larger fraction of an incoming DC beam than would be achievable with a single cavity; this is particularly important for spin-polarized operation where the amount of charge that can be extracted during the cathode lifetime is limited. To characterize the longitudinal bunch profile in a minimally invasive fashion, an SPR diagnostic chamber is installed downstream of the buncher after a drift space of about $1.5 \mathrm{~m}$. This location corresponds to the beginning of the pre-accelerator in the case of MESA.

The diagnostic device is designed to generate and observe the coherent part of SPR [10]. In our experiment, the interesting range of bunch charges is between 0.1 and 


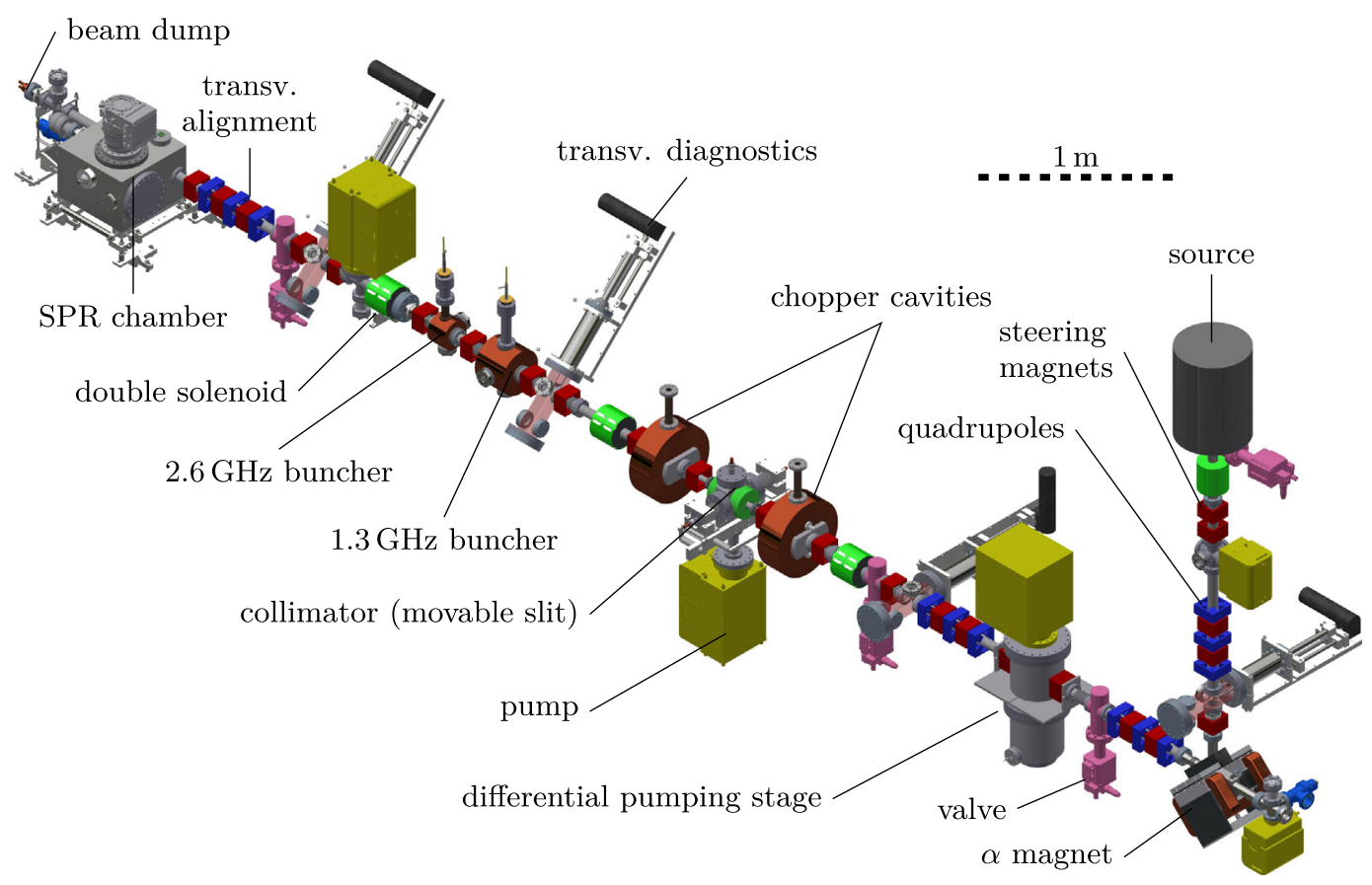

FIG. 3. Test apparatus for the injector of MESA. The DC electron beam from the source is chopped by two circularly deflecting rf cavities and an adjustable slit which cuts out a certain range of rf phases of the beam. Longitudinal compression is achieved with a system of two buncher cavities. Bunch length measurements are done in the SPR chamber. A quadrupole triplet including correction dipoles in front of the chamber provides transverse alignment of the beam.

$10 \mathrm{pC}$. Since the coherent fraction scales with the square of the bunch charge, the coherent/incoherent ratio increases with bunch charge. Due to $\mathrm{cw}$ operation, the average power level is sufficiently large to be detected with our detector $(>2 \mathrm{pW})$ even for the lowest charge. To obtain information on the bunch shape, a spectrally resolved measurement is needed. To keep the apparatus simple, the SPR observation angle is fixed and gratings with varying periods are introduced to obtain spectral information on the coherent part. Table I summarizes the parameters of this experiment.

\section{BUNCHER SYSTEM}

As the beam enters the buncher system at a nonrelativistic velocity of $\beta c$ with $\beta=0.548$, the longitudinal compression of the bunches is done using a phase-

TABLE I. Parameters of the experiment.

\begin{tabular}{lc}
\hline \hline Bunch repetition rate & $1.3 \mathrm{GHz}$ \\
\hline Beam energy & $100 \mathrm{keV}$ \\
$\beta$ & 0.548 \\
Bunch charge & $0.1 \mathrm{pC}$ to $2.3 \mathrm{pC}$ \\
Power SPR & $2 \mathrm{pW}$ to $50 \mathrm{nW}$ \\
Wavelength SPR & $\approx 1 \mathrm{~mm}$ \\
\hline \hline
\end{tabular}

dependent velocity modulation $\Delta \beta$. For long drift spaces between the buncher cavities and the focal point $\left(L_{f} \gg \beta \lambda_{\mathrm{RF}}\right)$, the needed modulation $\Delta \beta$ is linear with respect to the entrance phase of the reference particle in the first buncher cavity $\phi_{1}$.

$$
\Delta \beta \approx \frac{\beta^{2}}{2 \pi L_{f}} \cdot \phi_{1}
$$

Such a linear velocity modulation can be achieved using a first- and second-harmonic buncher cavity. The system of two buncher cavities described in [9] was adapted to the fundamental $\mathrm{rf}$ frequency of $1.3 \mathrm{GHz}$ of MESA (see [11]). The setup is shown in Fig. 4. With this arrangement, four free parameters have to be adjusted in order to obtain the best bunching result: the power in each cavity $P_{1}$ and $P_{2}$ and the corresponding phases $\phi_{1}$ and $\phi_{2}$ relative to the phase of the chopper cavities.

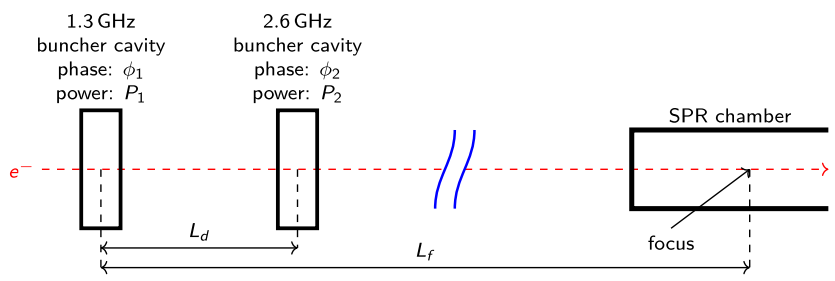

FIG. 4. Principle of longitudinal bunch compression using two cavities. 


\section{SPR SETUP}

The SPR apparatus is installed downstream of the MELBA (see Fig. 3). Here, several different gratings can be placed next to the beam, and the detector is at a fixed position in order to keep the system simple (Fig. 5). This allows to get frequency-domain information about the bunch without the need to install potentially lossy and mechanically complicated angle-scanning THz optics. The SPR radiation leaves the vacuum system through a z-cut quartz window. The power of the signal is measured for each individual grating. If the grating period $D$ is in the

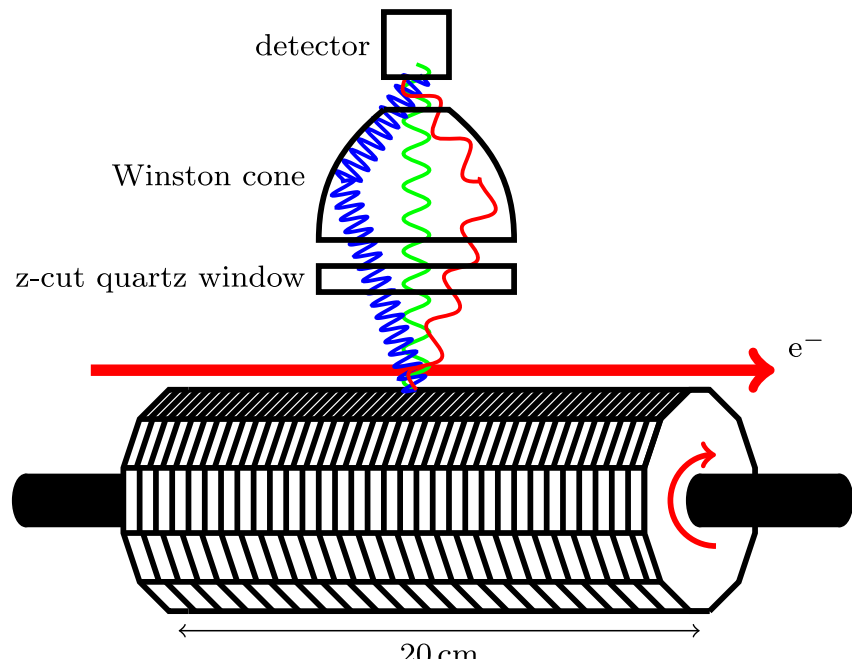

FIG. 5. Setup of the experiment. Multiple gratings can be placed next to the electron beam. The central observation angle is $\eta=\zeta=0^{\circ}$ with an angular spread of $\pm 2.1^{\circ}$. The generated $\mathrm{THz}$ radiation with a power of some $\mathrm{pW}$ to $30 \mathrm{nW}$ is observed with a bolometer cooled down to $4.2 \mathrm{~K}$.

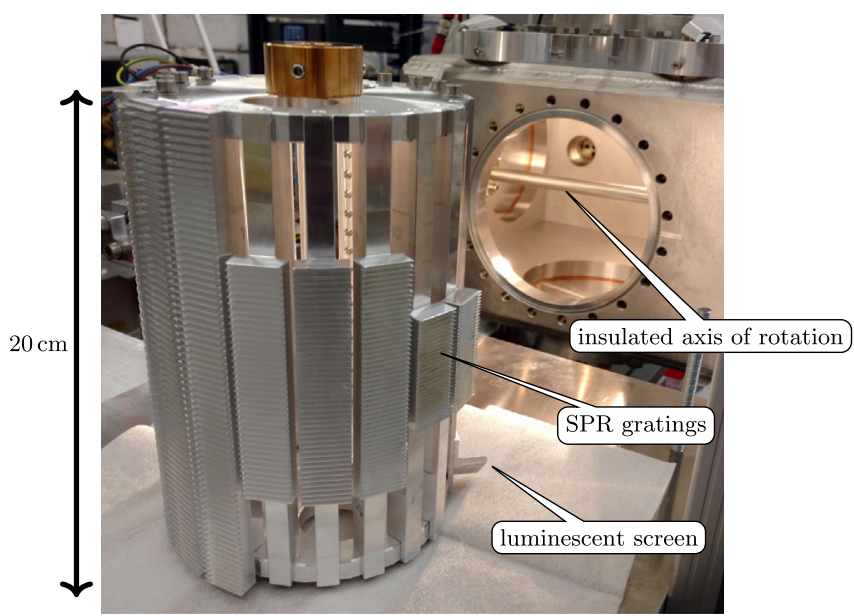

FIG. 6. Drum on which up to 20 gratings can be mounted $(0.75 \mathrm{~mm}<D<6 \mathrm{~mm})$. Transverse diagnostic tools (screens, holes, and wires) are contained as well. When installed in the SPR chamber, the drum is mounted on an insulated, rotatable axis.

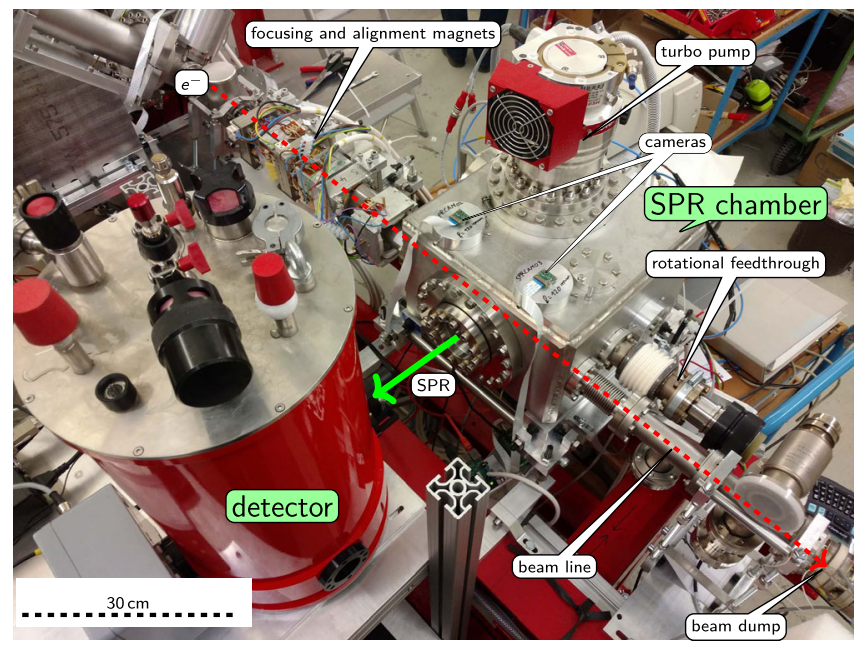

FIG. 7. Setup to detect SPR. The electron beam passes several magnets and is guided through the SPR chamber, in which the drum shown in Fig. 6 is mounted. The detector is placed directly in front of the vacuum window.

order of the bunch length, coherent enhancement of the SPR will increase the signal by a factor approaching $N_{e}$. The minimum period $D$ below which the coherent signal disappears is closely related to the bunch length, see Fig. 2.

Figure 6 shows a picture of the drum with different gratings mounted. The insulated axis makes it possible to measure the current of the small part of the electron beam which hits the grating. This simplifies the transverse alignment of the beam. Figure 7 shows the finished setup. The detector is a thermally sensitive resistance cooled down to $4.2 \mathrm{~K}$, which makes it possible to detect $\mathrm{THz}$ radiation with a power of $2 \mathrm{pW}$ at an integration time of $30 \mathrm{~s}$ and a signal-to-noise ratio of one. The red cryostat which cools the resistance is moved as close as possible to the vacuum window in order to reduce losses of $\mathrm{THz}$ radiation in air.

\section{MEASUREMENTS}

\section{A. Adjusting the system of buncher cavities}

In order to obtain short electron bunches at the location of the SPR grating, the four parameters of the buncher system $\left(P_{1}, P_{2}, \phi_{1}\right.$ and $\left.\phi_{2}\right)$ have to be adjusted. This is achieved using the grating with the largest period $(D=6 \mathrm{~mm})$. In the first measurement (Fig. 8), only the first-harmonic buncher cavity is turned on in order to reduce the number of free parameters to one phase $\phi_{1}$ and one rf power $P_{1}$. A maximum of the emitted SPR can be observed at a phase of $\phi_{1} \approx 270^{\circ}$. The value for $P_{1}=15 \mathrm{~W}$ is estimated from electromagnetic particle tracking simulations and a known shunt impedance of the cavity. In order to check if the signal results from coherent interaction, the bunch charge $Q_{\mathrm{b}}$ is increased at $\phi_{1}=270^{\circ}$ and the rise of the signal is measured. Looking at Eq. (2), the SPR signal 


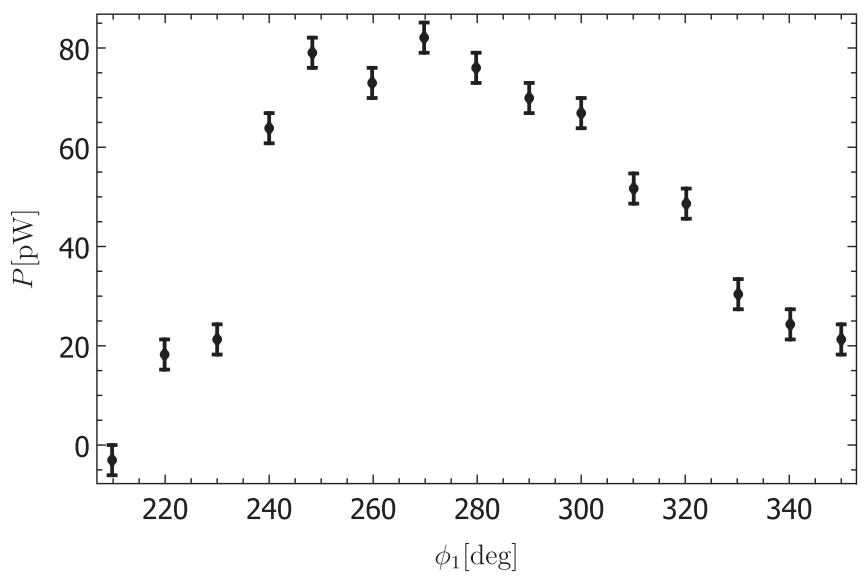

FIG. 8. SPR power $P$ to the phase of the first-harmonic buncher cavity $\phi_{1}$ at a fixed value of $P_{1}=15 \mathrm{~W}$. The second buncher cavity is turned off.

should rise with the square of the number of electrons per bunch $N_{e}$.

$$
\frac{\mathrm{d} P}{\mathrm{~d} \Omega} \propto N_{e}^{2} \cdot S_{\mathrm{coh}}
$$

Here, the beam current $I=Q_{\mathrm{b}} f=N_{e} f e$ is expressed in terms of electrons per bunch with the repetition rate of MESA of $f=1.3 \mathrm{GHz}$ and the elementary charge $e$. Figure 9 shows the measured behavior of the detected SPR power $P$ for different bunch charges $Q_{\mathrm{b}}$ or the number of electrons per bunch $N_{e}$, respectively. Here, $Q_{\mathrm{b}}$ is changed by variation of the power of the laser hitting the photocathode. A rise of the detected signal with respect to the square of the bunch charge can be observed. This means that the bunch length $\sigma_{x}$ is smaller than roughly

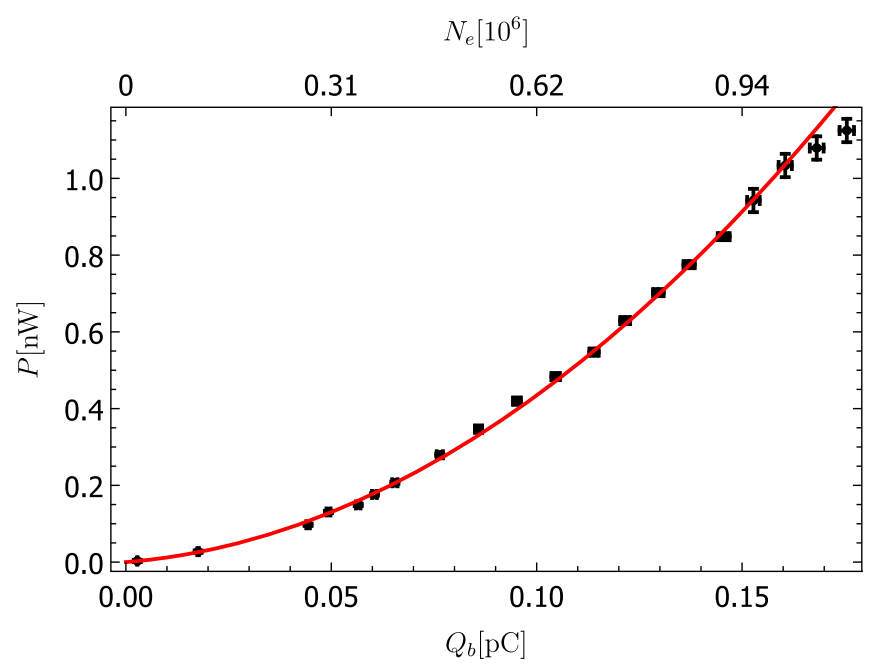

FIG. 9. SPR power $P$ for different bunch charges $Q_{\mathrm{b}}$. A rise of $P$ as the square of $Q_{\mathrm{b}}$ can be observed, proving that a coherent effect is detected.

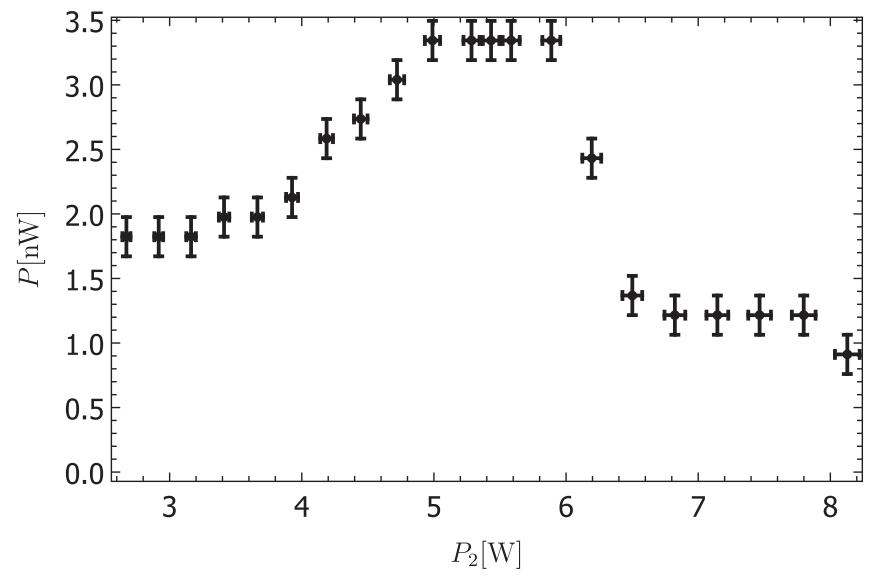

FIG. 10. SPR power $P$ vs. power of the second-harmonic buncher cavity $P_{2}$ at the smallest grating period $D=0.75 \mathrm{~mm}$.

$D / 2=3 \mathrm{~mm}$. Other, potentially coherent, background radiation can be excluded (see Sec. VID).

The method described above is repeated for smaller grating periods $D$ until the best set of parameters $\left(P_{1}, P_{2}\right.$, $\phi_{1}$ and $\left.\phi_{2}\right)$ is found at the smallest $D=0.75 \mathrm{~mm}$. Figure 10 shows an example of the measured SPR signal $P$ for different powers of the second-harmonic buncher cavity $P_{2}$. A maximum of the emitted SPR can be observed at $P_{2} \approx 5.5 \mathrm{~W}$. In analogy to Fig. 9 , a variation of the bunch charge leads to a quadratic rise of the signal. Therefore, the bunch length must be smaller than $\sigma_{x} \lesssim D / 2=375 \mu \mathrm{m}$, and the optimum parameters of the buncher system are $P_{1}=15.2 \mathrm{~W}$ and $P_{2}=5.4 \mathrm{~W}$. The phases $\phi_{1}$ and $\phi_{2}$ do not contain relevant information because they depend on experiment-specific parameters such as cable length. Electromagnetic simulations have shown that it is possible to operate the preaccelerator for MESA (MAMBO [12]) with an energy width of $\Delta E_{\mathrm{RMS} \text {,design }}=1.022 \mathrm{keV}$ and a bunch length of $\sigma_{x \text {,design }}=1.7 \mathrm{~mm}$ at the entrance of the first acceleration section [13]. The measured bunch length of $\sigma_{x} \lesssim 375 \mu \mathrm{m}$ and the produced energy width with the set parameters $P_{1}$ und $P_{2}$ of $\Delta E_{\mathrm{RMS}} \approx 1 \mathrm{keV}$ therefore exceeds the requirements.

\section{B. Further bunch length investigation}

Until now, only an upper limit for the bunch length has been determined. In order to obtain a more precise value of $\sigma_{x}$, a comparison of the calculated SPR power with the measured one can be done. For the calculation of the emitted SPR power in particular, all beam parameters entering Eq. (2) and Eq. (3) have to be known. The transverse beam profile is measured with luminescent screens, and the beam current can be determined using a Faraday cup (here beam dump). Additionally the reflection coefficient $\left|R_{n}\right|^{2}$ has to be calculated (see Sec. VIC). Figure 11 shows a measurement in which the distance between beam and grating $z_{0}$ (see Fig. 1) is varied. With this method, a fixed value for the bunch length of $\sigma_{x}=$ 


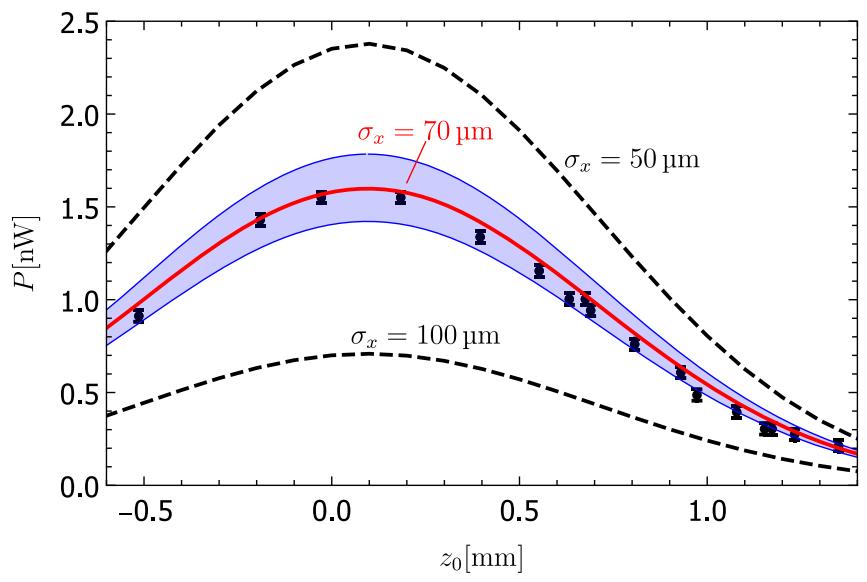

FIG. 11. SPR power $P$ vs. distance between beam and grating $z_{0}$. Red curve: Calculated behavior [Eq. (2)] with corresponding error (blue) for $Q_{\mathrm{b}}=(270 \pm 10) \mathrm{fC}, \sigma_{z}=\sigma_{y}=(866 \pm 15) \mu \mathrm{m}$ and a bunch length of $\sigma_{x}=(70 \pm 5) \mu \mathrm{m}$. The dotted lines indicate the calculated behavior for $\sigma_{x}=50 \mu \mathrm{m}$ and $\sigma_{x}=100 \mu \mathrm{m}$.

$(70 \pm 5) \mu \mathrm{m}$ can be determined. In this calculation, an error of the reflection coefficient of $\Delta\left|R_{n}\right|^{2}= \pm 20 \%$ is assumed (see Sec. VIC).

\section{Reflection coefficient}

Referring to Eq. (2), the emitted SPR is proportional to the reflection coefficient $\left|R_{n}\right|^{2}=\left|R_{n}\right|^{2}(n, \beta, \eta, \zeta, a / D, h / D)$, where $a$ and $d$ describe the geometry of the respective grating (see Fig. 12). It is possible to calculate $\left|R_{n}\right|^{2}$ for rectangular gratings numerically with the approach of van den Berg. In this experiment five different geometries are chosen. Note that gratings with different periods $D$ can have the same fractions $a / D, h / D$ and thus the same reflection coefficient. Table II shows the values of the calculated reflection coefficients for the observation angle of $\eta=\zeta=0^{\circ}$. In order to get a strong signal, a high value of the reflection coefficient is favorable.

The errors of the coefficients are estimated in a dedicated experiment: Gratings with the same period $D$ but with different geometries are successively placed next to the electron beam without changing any beam parameters. The measured SPR powers are then compared and the deviation from its theoretical behavior is investigated.

One has to note that multiple radiation orders $n$ are always observed at the same time by the detector. A more precise analysis of the reflection coefficient at a specific order of radiation $n$ was done by using a multiwire grid band-pass filter which is placed between the grating and the

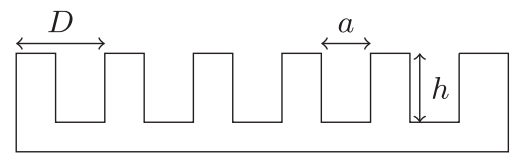

FIG. 12. Geometry of the gratings.
TABLE II. Reflection coefficients for $\eta=\zeta=0^{\circ}$ and $\beta=$ 0.548 in the first three radiation orders $n$ and different geometries.

\begin{tabular}{ccccc}
\hline \hline$a / D$ & $h / D$ & $\left|R_{1}\right|^{2}$ & $\left|R_{2}\right|^{2}$ & $\left|R_{3}\right|^{2}$ \\
\hline 0.80 & 0.92 & $0.4 \times 10^{-3}$ & $14 \times 10^{-3}$ & $0.1 \times 10^{-3}$ \\
0.27 & 0.57 & 0.35 & $47 \times 10^{-3}$ & $0.86 \times 10^{-3}$ \\
0.33 & 1.04 & $83 \times 10^{-3}$ & $95 \times 10^{-3}$ & $8.8 \times 10^{-3}$ \\
0.35 & 1.66 & $80 \times 10^{-3}$ & $1.9 \times 10^{-3}$ & $2.1 \times 10^{-3}$ \\
0.26 & 1.80 & $2.1 \times 10^{-3}$ & $11 \times 10^{-3}$ & $4.4 \times 10^{-3}$ \\
\hline \hline
\end{tabular}

detector. This filter is chosen in such a way that only one order of radiation $n$ of the SPR is transmitted.

With these investigations, an error for the reflection could be estimated: In sum over all observable radiation orders $n$, the reflection coefficients are assumed to have an error of roughly $20 \%$. As a preliminary result, a suppression of high values for the reflection coefficients could be observed. Especially the high value of 0.35 in Table II could be disproved. At this point, further investigations with more band-pass filters and an experimental determination of the coefficients with known bunch length are needed to discuss this problem in an adequate manner.

\section{SPR as a minimally invasive measurement tool}

As seen in Fig. 11, the SPR signal $P$ decreases fast with growing distance between beam and grating $z_{0}$. Nevertheless, a signal can be observed at high values of $z_{0}$. In the following measurements, the transverse shape of the beam is not changed. In Fig. 13, the bunch charge $Q_{\mathrm{b}}$ is varied for five different distances between beam and grating. The SPR signal rises with the square of $Q_{\mathrm{b}}$, as observed in Fig. 9. Therefore, the signal must be coherent, and bunch length measurements can be done while losing less than $0.6 \%$ of the beam in the case of $z_{0} / \sigma_{z}=2.54$. The dashed curves describe a quadratic fit of the data:

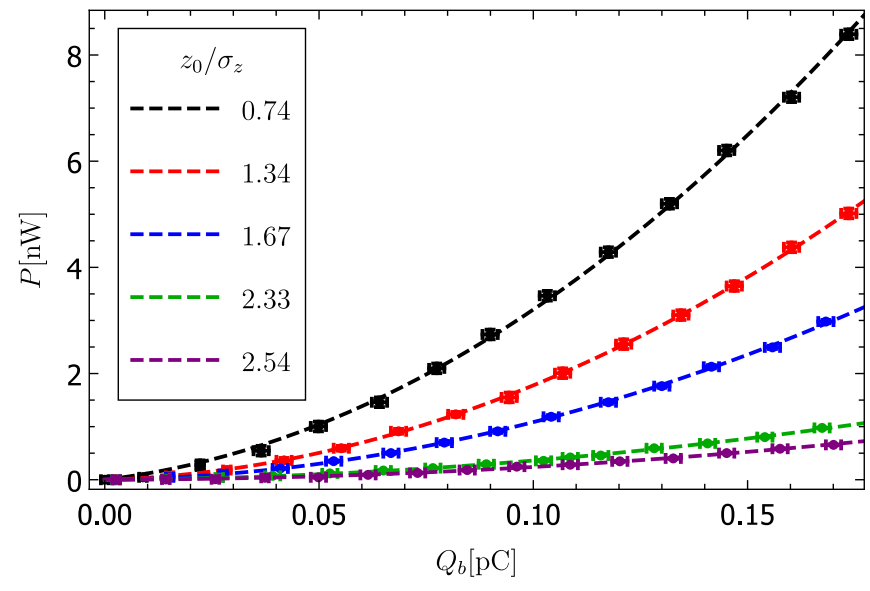

FIG. 13. SPR power $P$ to bunch charge $Q_{\mathrm{b}}$ for different distances between beam and grating $z_{0}$. The transverse size of the beam $\sigma_{z} \approx 100 \mu \mathrm{m}$ is not changed. 
TABLE III. Fit parameters of the dashed curves in Fig. 13.

\begin{tabular}{lccc}
\hline \hline$z_{0} / \sigma_{z}$ & $a[\mathrm{nW} / \mathrm{pC}]$ & $b\left[\mathrm{nW} / \mathrm{pC}^{2}\right]$ & $a / b\left[10^{-3} \mathrm{pC}\right]$ \\
\hline $0.74 \pm 0.04$ & $9.81 \pm 1.41$ & $223 \pm 11$ & $44.0 \pm 6.7$ \\
$1.34 \pm 0.02$ & $2.38 \pm 0.93$ & $154 \pm 7$ & $15.5 \pm 6.1$ \\
$1.67 \pm 0.02$ & $1.08 \pm 0.46$ & $97.4 \pm 3.9$ & $11.1 \pm 4.8$ \\
$2.33 \pm 0.07$ & $0.46 \pm 0.15$ & $31.4 \pm 1.3$ & $14.6 \pm 4.9$ \\
$2.54 \pm 0.06$ & $0.22 \pm 0.12$ & $21.8 \pm 0.9$ & $10.1 \pm 5.7$ \\
\hline \hline
\end{tabular}

$$
P=a \cdot Q_{\mathrm{b}}+b \cdot Q_{\mathrm{b}}^{2}
$$

The parameters $a$ and $b$ are listed in Tab. III. The fraction $a / b$ decreases with growing distance $z_{0}$. This means that linear effects are less significant at larger distances between beam and grating. Fig. 14 shows the linear part of the signal $a$ for the five set distances $z_{0} / \sigma_{z}$. The linear part of the signal $a$ can be explained by calculating how many electrons are getting lost on the grating. The transverse particle distribution $\left[f_{z}(z)\right.$ in Eq. (3)] is known. Integrating this distribution up to the surface of the grating $\left(z_{0}=0\right)$ yields the red curve in Fig. 14 with the corresponding error band in blue. This means that particles which hit the grating produce a linear background signal, and the coherent part of the signal is only caused by electrons which do not hit the grating. This property of the observed radiation can only be described by the underlying mechanism of SPR.

\section{E. Space charge effects}

Coulomb interactions between the electrons lead to a blow-up of the particle distribution at higher bunch charges. In Fig. 15, the bunch charge is increased up to $Q_{\mathrm{b}} \approx$ $0.75 \mathrm{pC}$ and the bunch length $\sigma_{x}$ is determined by comparing the calculated and measured SPR power. The black curve shows the behavior for the fixed values for the buncher system $\left(P_{1}, P_{2}, \phi_{1}\right.$ and $\left.\phi_{2}\right)$ described in Sec. VI A. In this measurement, space charge mostly affects the

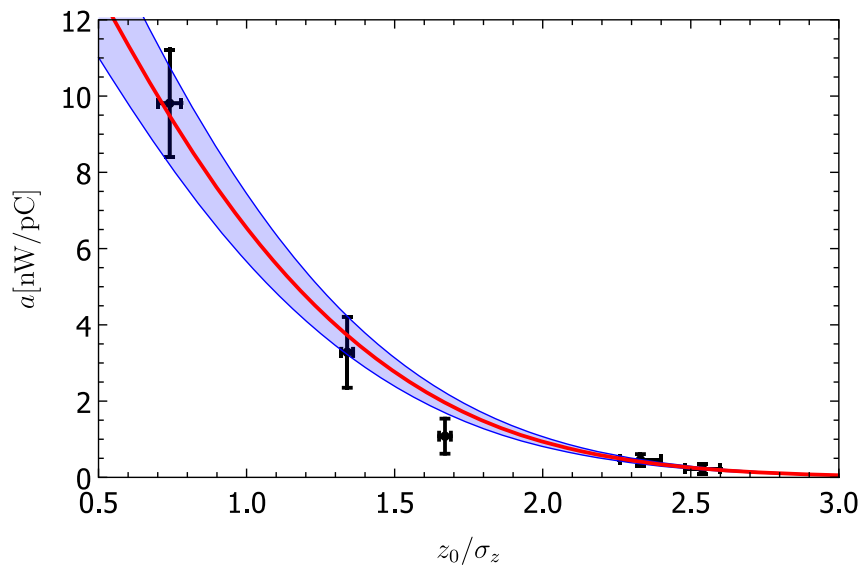

FIG. 14. Linear part of the SPR signal $a$ shown in Fig. 13 for the five different applied distances (values from Table III).

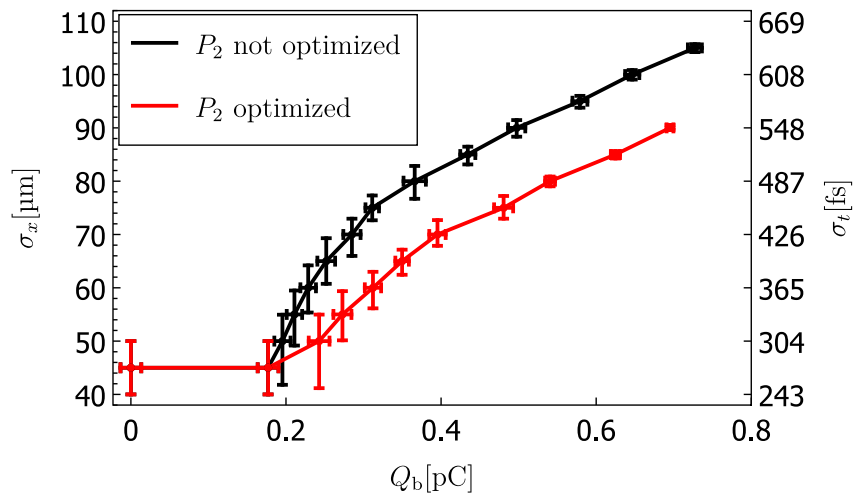

FIG. 15. Bunch length $\sigma_{x}$ to bunch charge $Q_{\mathrm{b}}$. The power of the second-harmonic buncher cavity $P_{2}$ is optimized for different bunch charges.

longitudinal particle distribution. Simulations have shown that the relative increase of the transverse beam size (here $\sigma_{y}=\sigma_{z} \approx 870 \mu \mathrm{m}$ ) due to space charge is about $15 \%$. This effect can be neglected here because the change in the emitted SPR power $P$ at a variation of $\Delta \sigma_{z}= \pm 15 \%$ is low. In Fig. 15, the impact of space charge on the longitudinal particle distribution can be observed starting at bunch charges of $Q_{\mathrm{b}} \approx 0.2 \mathrm{pC}$. At smaller $Q_{\mathrm{b}}$, no change in $\sigma_{x}$ can be observed within the resolution of our detection system. Thus, the nonlinear effect of the space charge has to be considered only for $Q_{\mathrm{b}}>0.2 \mathrm{pC}$ at a transverse beam size of $\sigma_{z} \approx 870 \mu \mathrm{m}$. At this point, the electron density becomes high enough for the Debye-length [14] to be small compared to the spacial dimensions of the bunch.

Considering nonlinear effects, the best velocity modulation is not necessarily linear as assumed in Eq. (5). So the increase in $\sigma_{x}$ can be compensated for to a certain point by re-adjusting the four parameters of the buncher system in order to obtain a better, yet unknown, velocity modulation. For example, a variation of the power of the secondharmonic buncher cavity $P_{2}$ is adjusted for bunch charges greater than $0.2 \mathrm{pC}$ (red curve in Fig. 15). Here, a shortening of the bunch length $\sigma_{x}$ by approximately $10 \%$ can be achieved by increasing the power $P_{2}$ from $5.4 \mathrm{~W}$ at low bunch charges to $8.2 \mathrm{~W}$ at $Q_{\mathrm{b}}=0.75 \mathrm{pC}$.

\section{LIMITATION OF BUNCH LENGTH DETERMINATION}

The bunch length measuring limit with the method described is defined by the grating period $D$. If $\sigma_{x} \ll D$, the coherent enhancement factor $S_{\text {coh }}$ (see Fig. 2) saturates and changes in the bunch length cannot be resolved by the detection system. Figure 16 shows the calculated SPR power for short bunch lengths at the smallest available grating of $D=0.75 \mathrm{~mm}$. So the available detection system provides a bunch length measuring limit of roughly $10 \mu \mathrm{m}$ at a grating with $D=0.75 \mathrm{~mm}$. 


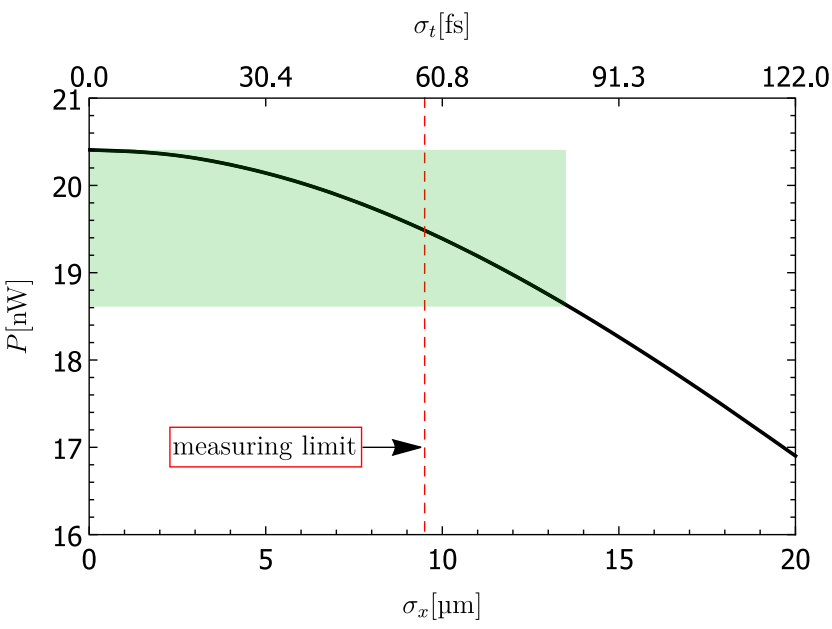

FIG. 16. Calculated SPR power for short bunch lengths at the smallest available grating period $D=0.75 \mathrm{~mm}$. At a bunch length of $\sigma_{x} \lesssim 10 \mu \mathrm{m}$, changes in $\sigma_{x}$ cannot be resolved by the detection system. The green box indicates the measuring accuracy of the detection system of $\pm 4.4 \%$.

\section{CONCLUSION}

It was shown that coherent Smith-Purcell radiation can be used to measure bunch lengths at a test apparatus for the MESA accelerator. It is an minimally invasive method whereby less than $0.6 \%$ of the beam is lost. This makes it possible to do live measurements even at high beam currents. The observation of a coherent effect at a grating with known constant $D$ defines an upper limit of the bunch length. More precise results can be obtained by comparing the measured data with theory.

The achievable bunch length makes it possible to operate $M E S A$ with bunch charges of $0.75 \mathrm{pC}$, which essentially fulfills the specifications for the operation of MESA in its first stage. In addition, there are other conceivable applications of the measurement method: Ultrafast processes on surfaces can be observed with time-resolved electron microscopes. Therefore, short electron pulses of about $100 \mathrm{fs}$ are needed with typical energies of $1 \mathrm{keV}$ to $100 \mathrm{keV}$, which is in the same order of magnitude as the experiment carried out.

For future measurements, it would be favorable to use calibrated SPR gratings. To obtain these, an alternative invasive bunch length measuring method using a deflecting cavity and a luminescent screen can be used. The SPR power is then measured with the knowledge of all beam parameters. By using band-pass filters, each radiation order $n$ can be observed separately. With this method, the reflection coefficient $\left|R_{n}\right|^{2}$ can be determined with better accuracy and for all orders $n$. Such a calibrated grating could then be used to obtain even more precise results.
Depending on the bunch charge, other detector systems without the need to be cooled to $4 \mathrm{~K}$ can be used if the signal is strong enough. Then, a more compact design of the whole apparatus can be applied even at space-limited machines.

\section{ACKNOWLEDGMENTS}

This project was funded by the German Federal Ministry of Education and Research (BMBF) under No. 05K19UMA "Subpikosekunden spinpolarisierte Bunche und Bunchlängen-Messung mit Smith Purcell Strahlung". The Mainz cluster of excellence PRISMA, Precision Physics, Fundamental Interactions and Structure of Matter, is also greatly acknowledged for financially bridging this project. The authors gratefully acknowledge the strong support by the mechanical and electronic workshops at the institute for nuclear physics at JGU Mainz. In particular, we thank Dr. W. Lauth (Head of electronics laboratory) for his help and valuable contributions.

[1] P. M. van den Berg, J. Opt. Soc. Am. 63, 1588 (1973).

[2] P. M. van den Berg and T. H. Tan, J. Opt. Soc. Am. 64, 325 (1974).

[3] S. J. Smith and E. M. Purcell, Phys. Rev. 92, 1069 (1953).

[4] B. Hua and D. Zhi-Min, Chin. Phys. C 32, 916 (2008).

[5] C. Matejcek, Strahldynamik der Niederenergie-Strahlführung von MESA unter Berücksichtigung von Raumladung und Multipol-Beiträgen höherer Ordnung, Ph.D. thesis, Johannes Gutenberg-Universität Mainz, 2020.

[6] S. Friederich, Entwicklung einer hochbrillanten Photoemissionsquelle für spinpolarisierte Strahlen, Ph.D. thesis, Johannes Gutenberg-Universität Mainz, 2019.

[7] P. Heil, Kohärente Smith-Purcell-Strahlung zur minimal invasiven Bunchlängenmessung im Subpikosekundenbereich, Ph.D. thesis, Johannes Gutenberg-Universität Mainz, 2020.

[8] S. Friederich, K. Aulenbacher, and C. Matejcek, in Proceedings of the 10th Int. Particle Accelerator Conf. IPAC2019, Melbourne, Australia (JACoW, Geneva, 2019).

[9] V. I. Shvedunov et al., Conf. Proc. C 960610, 1556 (1996).

[10] G. Doucas, M. F. Kimmitt, A. Doria, G. P. Gallerano, E. Giovenale, G. Messina, H. L. Andrews, and J. H. Brownell, Phys. Rev. Accel. Beams 5, 072802 (2002).

[11] P. Heil, Longitudinale Emittanzanpassung durch Geschwindigkeitsmodulation im Injektionssystem an MESA, Master's thesis, Johannes Gutenberg-Universität Mainz, 2015.

[12] F. Hug and R. Heine, J. Phys. Conf. Ser. 874, 012012 (2017).

[13] R. Heine, Phys. Rev. Accel. Beams 24, 011602 (2021).

[14] T. P. Wangler, RF Linear Accelerators (Wiley-VCH $\mathrm{GmbH}$, New York, 2008). 